\title{
Recent results on structure functions
}

\section{Haiyan Gao*}

Department of Physics, Duke University and Triangle Universities Nuclear Laboratory, Durham, NC 27708, U.S.A.

E-mail: gao@phy.duke.edu

Significant progress has been made in recent years in both unpolarized structure functions of the nucleon as well as polarized structure functions. More recently, both experimental and theoretical studies of the structure of the nucleon have advanced beyond the one-dimensional collinear approximation, and three-dimensional descriptions of the nucleon structure both in momentum space, and longitudinal momentum-transverse space are becoming active areas of research. In this talk, I will review recent results on structure functions of the nucleon.

35th International Conference of High Energy Physics - ICHEP2010,

July 22-28, 2010

Paris France

\footnotetext{
*Speaker.
} 


\section{Introduction}

Strong interaction is one of the corner stones of the Standard Model of particle physics. Quantum chromodynamics (QCD) is the accepted theory of strong interaction with quarks and gluons as the fundamental degrees of freedom. Asymptotic freedom is an important and unique feature of QCD which allows for robust tests of the theory in the high energy regime where perturbative QCD calculations can be carried out. However, in the confinement region, where ordinary matter lives, perturbative calculations are invalid due to the large strong coupling constant. In the absence of an analytical solution of the QCD Lagrangian, little is known about how QCD really works in the non-perturbative region. Nucleons are fundamental building blocks of matter, and the structure of which provides insight and foundation to understand how QCD works. As such the study of the nucleon structure has become an ever more active and important area of research. Significantly improved knowledge about the structure of the nucleon in terms of QCD is also essential for new physics beyond the Standard Model at high energy frontiers because strong interaction processes are often important physics backgrounds. The rest of this paper is organized as following. Section II discusses recent progress in the study of unpolarized proton structure functions followed by advances in the study of polarized structure functions of the nucleon. In Section IV, I will discuss generalized parton distribution functions (GPDs) and transverse-momentum-dependent parton distribution functions (TMDs).

\section{Unpolarized structure functions}

Deep inelastic lepton-nucleon scattering (DIS) experiments have played a fundamental role in describing the partonic momentum structure of hadrons. The unpolarized parton distribution functions (PDF) have been extracted with excellent precision over a large range of $x$ and $Q^{2}$ from DIS, Drell-Yan and other processes after several decades of experimental and theoretical efforts. The comparison of the structure functions in the large $Q^{2}$ range with QCD evolution equations has provided one of the best tests of QCD.

Recently, a combined analysis [1] of inclusive neutral current and charged current unpolarized $\mathrm{e}^{ \pm} \mathrm{p}$ collision from HERA for data collected from 1994 to 2000 has been released by the $\mathrm{H} 1$ and the ZEUS Collaborations. These data cover six orders of magnitude in negative four-momentumtransfer-squared, $Q^{2}$, and Bjorken $x$. New parton distribution functions with smaller experimental uncertainties based on HERA data only, HERAPDF1.0, are now available following the NLO QCD analysis. Fig. 1 shows the combined HERA neutral current $\mathrm{e}^{+} \mathrm{p}$ reduced cross section data (solid circles) together with fixed-target data (open squares). The HERAPDF1.0 fit together with total uncertainty of the fit is superimposed (blue band). Fig. 2 shows parton distribution functions from HERAPDF1.0 together with the experimental, model and parametrization uncertainties. These new precision data are particularly important for interpreting new results from LHC.

\section{Polarized structure functions}

The term "spin crisis" originated from the experimental discovery by the European Muon Collaboration (EMC) experiment in the 1980s [2] that quark spin seems to contribute only about 


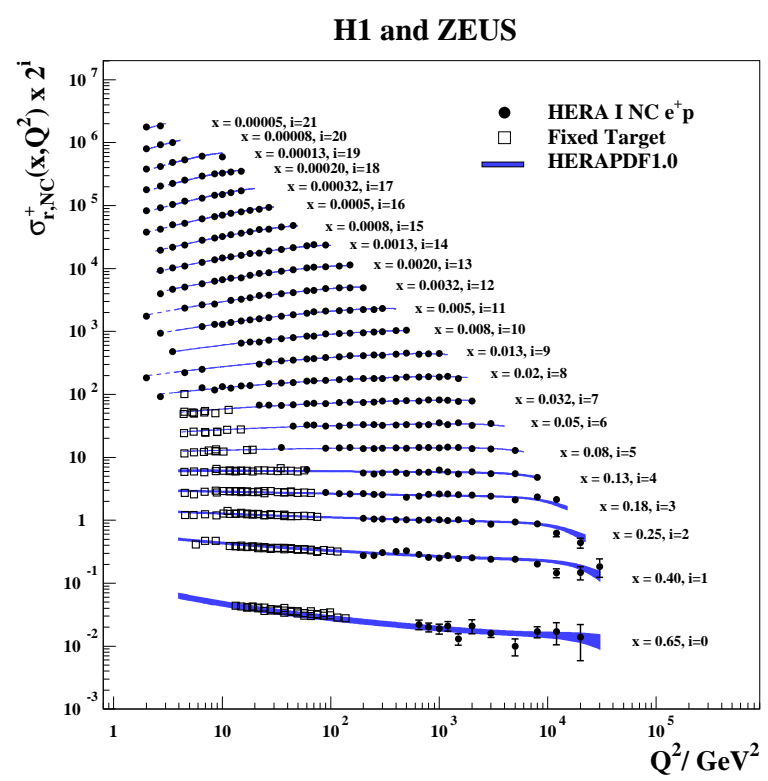

Figure 1: (From Fig. 9 of [1]) HERA combined $\mathrm{NC} \mathrm{e}^{+} \mathrm{p}$ reduced cross section and fixed-target data as a function of $Q^{2}$. The error bars indicate the total experimental uncertainty. The HERAPDF1.0 fit is superimposed. The bands represent the total uncertainty of the fit. Dashed lines are shown for $Q^{2}$ values not included in the QCD analysis.
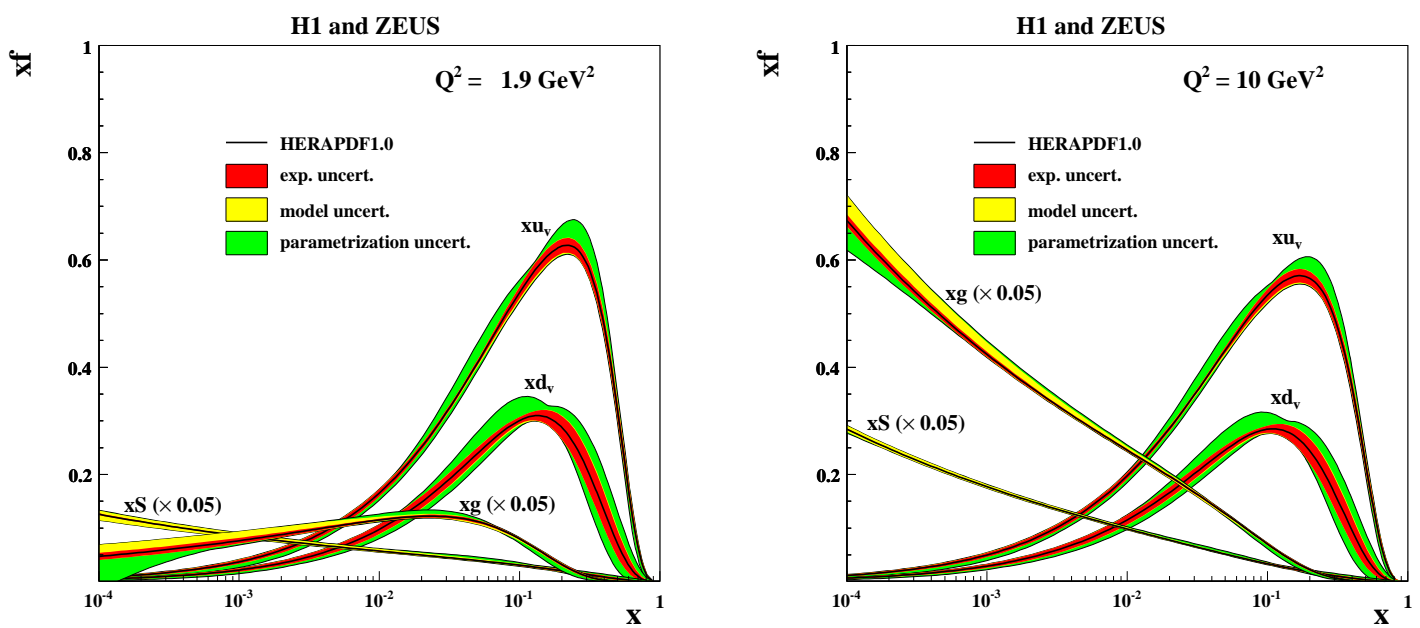

Figure 2: (From Fig. 18 of [1]) The parton distribution functions from HERAPDF1.0, $x u_{v}, x d_{v}, x S=$ $2 x(\tilde{U}+\tilde{D}), x g$, at $Q^{2}=1.9 \mathrm{GeV}^{2}$ (left) and $Q^{2}=10 \mathrm{GeV}^{2}$ (right). The gluon and sea distributions are scaled down by a factor 20 . The experimental, model and parametrization uncertainties are shown separately.

one third of the total spin of the nucleon. Motivated by the EMC result, three decades of intensive experimental and theoretical investigation have resulted in a great deal of knowledge on the partonic 
origin of the nucleon spin structure. This is especially the case with the longitudinal polarized parton distribution functions, which in the parton model, are the probabilities of partons polarized in the longitudinal polarization direction of the nucleon. The nucleon spin can be written in the following way:

$$
\frac{1}{2}=\frac{1}{2} \Delta \Sigma+\Delta G+L
$$

where $\Delta \Sigma$ and $\Delta G$ are the corresponding quark spin and gluon spin contribution to the nucleon spin, and $L$ is the total angular momentum contribution from quarks and gluons to the nucleon spin. The longitudinal parton distribution functions, which determine $\Delta \Sigma$, have been determined with significantly improved precision over a large range of $x$ and $Q^{2}$ from polarized DIS experiments at CERN, SLAC, DESY in the last two decades, and more recently at JLab and at RHIC from polarized proton-proton scattering (see $[3,4]$ for reviews and compilation of references). These more precise measurements confirm the original EMC observation. The latest result on the quark spin contribution to the nucleon spin, $\Delta \Sigma=0.32 \pm 0.03$ (stat.), is from the COMPASS analysis [5] of semi-inclusive deep-inelastic scattering of longitudinally polarized muons from polarized $\mathrm{NH}_{3}$ target and $\mathrm{ND}_{3}$ target. This result is also consistent with the 2007 NLO QCD fit by the COMPASS collaboration [6] using all the world data available at the time.

In more recent years, significant efforts have been devoted to the study of the gluon spin contribution to the spin of the nucleon both through double-polarized DIS and double polarized proton-proton scattering. In DIS, the renormalization and factorization scale, $\mu^{2}$ is set to be equal to $Q^{2}$. Since virtual photons do not couple directly to gluons, sensitivity to gluon helicity distribution through DGLAP evolution of parton longitudinal spin distribution functions is rather weak at leading order. At next-to-leading order, additional sensitivity to gluon polarization is through photon-gluon fusion subprocess identified by open charm production or through selecting high $P_{T}$ hadron pair in the final state in DIS. Both approaches have been pursued by the HERMES experiment, and the COMPASS experiment in polarized DIS measurements with double polarizations. Figure 3 (Fig.19 of [7]) shows results on $\frac{\delta G}{G}$ from polarized DIS experiments. The Hermes result on high $P_{T}$ hadrons $\left(1.0<P_{T}<2.5 \mathrm{GeV} / \mathrm{c}\right)$ from deuteron target is shown as the red solid point. The red solid line is the HERMES fct- 1 fit in the $x$ range of the HERMES data only. Details can be found in Ref. [7]. The COMPASS result from Open Charm [8] is shown as black solid triangle. Results from high $P_{T}$ pairs from SMC [9], COMPASS low $Q^{2}$ [10], and COMPASS high $Q^{2}$ [11] are shown as open circle, solid and open square, respectively. These results suggest that the gluon spin contribution to the spin of the nucleon is small, which are consistent with results from polarized proton-proton scattering at RHIC [12, 13]. Also shown in Fig. 3 are NLO pQCD fit DSSV [14] which includes world data on inclusive, semi-inclusive DIS and polarized proton-proton collision, and BB-09 [15] fit including inclusive DIS data only, both at a scale of $\mu^{2}=1.5 \mathrm{GeV}^{2}$.

\section{Three-dimensional descriptions of the structure of the nucleon}

In more recent experimental and theoretical studies, it has become evident that precise knowledge of the transverse structure in both momentum and impact parameter dependent distributions of partons is essential to unfold the full momentum and spin structure of the nucleon. While 


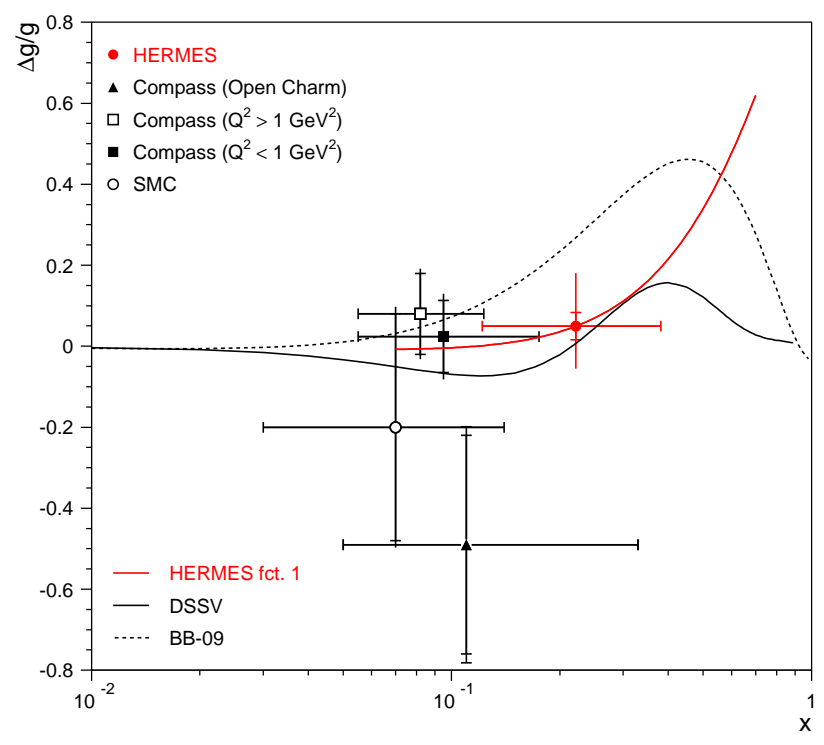

Figure 3: (From Fig. 19 of [7]) The gluon polarization $\frac{\Delta G}{G}$ from HERMES extracted with fct. $1(<x>=$ $0.22,<\mu^{2}>=1.35 \mathrm{GeV}^{2}$ ) compared to the ones from COMPASS [10, 11, 8] (low $Q^{2}: \mu^{2}=3 \mathrm{GeV}^{2}$, high $Q^{2}: \mu^{2}=2.4 \mathrm{GeV}^{2}$, open charm: $\left.\mu^{2}=13 \mathrm{GeV}^{2}\right)$ and SMC [9] $\left(\mu^{2}=3.6 \mathrm{GeV}^{2}\right)$ including statistical uncertainties (inner error bars) and total uncertainties (outer error bars). The $x$ region of the data is indicated by the horizontal bars. Fit function fct. 1 is shown over the full $x$ range spanned by the HERMES data. Also shown are a sample of curves from NLO pQCD fits DSSV, and BB-09 at $\mu^{2}=1.5 \mathrm{GeV}^{2}$. For clarity only the central values are shown.

the transverse momentum dependent parton distribution functions (TMDs) [16] provide threedimensional description of the structure of the nucleon, the generalized parton distribution functions (GPDs) [17] is another approach to provide a different three-dimensional mapping of the structure of the nucleon. In the latter case, the three dimensions involve one-dimensional longitudinal momentum space and two-dimensional transverse space. The formalism of GPDs is advantageous because it allows for connections of the elastic form factors of the nucleon, structure functions probed in deep-inelastic processes, and spin through GPDs. Furthermore, it allows for a direct experimental access to the quark orbital angular momentum inside the nucleon. The cleanest access to GPDs is through Deeply Virtual Compton Scattering (DVCS) $\left(\gamma^{*} N \rightarrow \gamma N\right)$, in which a virtual photon of high virtuality scatters off a nucleon and produces a real photon in the final state. There has been intensive experimental activities on DVCS. This includes the DVCS cross section measurements by H1 Collaboration [18, 19] and ZEUS Collaboration [20], the HERMES relative beam-helicity [21] and beam-charge asymmetry measurements [22, 23], Jefferson Lab (JLab) CLAS results on DVCS beam-asymmetry [24, 25] and target polarization observables [26], and JLab DVCS results on beam-helicity asymmetry from Hall A [27]. Another approach which allows for an access to GPDs and also a flavor separation is through Deeply Virtual Meson Production (DVMP) processes. However, the interpretation of the data [28, 29] in the frame work of GPDs is more complicated than that of DVCS. For a review of the subject of GPDs, we refer readers to papers in this conference proceedings by Dieter Müller and Michel Guidal. 
At leading twist if we integrate over the transverse momenta of quarks, the three quark distribution functions remain: the unpolarized parton distribution $f_{1}$, the longitudinal polarized parton distribution $g_{1}$, and the quark transversity distribution $h_{1}$ (denoted also as $\delta q(x)$, or $\Delta_{T} q(x)$ ). The transversity function is the least known among the three leading twist parton distribution functions. It is a chiral-odd transversely polarized quark distribution function [30] and describes the net quark transverse polarization in a transversely polarized nucleon [31]. In the non-relativistic limit, the transversity distribution function $h_{1}\left(x, Q^{2}\right)$ is the same as the longitudinal quark polarization distribution function, $g_{1}\left(x, Q^{2}\right)$. Like the axial charge $\Delta q^{a}=\int_{0}^{1} d x\left(g_{1}^{a}(x)+g_{1}^{-q}(x)\right)$, the tensor charge $\delta q^{a}=\int_{0}^{1} d x\left(h_{1}^{a}(x)-h^{\bar{q}}(x)\right)$ is a basic property of the nucleon.

Besides $f_{1}, g_{1}$ and $h_{1}$, there are five more transverse momentum dependent distribution functions $[32,33]$. These five functions are the Sivers function $\left(f_{1 T}^{\perp}\right)$, the Boer-Mulders function $\left(h_{1}^{\perp}\right)$, the pretzelocity function $\left(h_{1 T}^{\perp}\right)$, and the so-called worm-gear $1\left(h_{1 L}^{\perp}\right)$ and worm-gear $2\left(g_{1 T}\right)$ functions. Since these TMDs provide the description of the parton distributions beyond the collinear approximation, they depend not only on the longitudinal momentum fraction $x$, but also on the transverse momentum, $\overrightarrow{k_{T}}$. An intuitive interpretation of the $\overrightarrow{k_{T}}$ dependent transversity distribution, $h_{1}$, is that it gives the probability of finding a transversely polarized parton inside a transversely polarized nucleon with certain longitudinal momentum fraction $x$ and transverse momentum $\overrightarrow{k_{T}}$.

The experimental determination of the transversity function is challenging - it is not accessible in polarized inclusive DIS measurements due to its chiral-odd nature. However, paired with another hadron in the initial state, for example double polarized Drell-Yan processes with (two transversity distributions) [34], or in the final state, for example semi-inclusive deep-inelastic [35] scattering (transversity and Collins fragmentation function), leading twist $h_{1}$ can be accessed without suppression by a hard scale. The most feasible way to access the transversity distribution function is via an azimuthal single spin asymmetry, in semi-inclusive deep-inelastic lepto-production of mesons on a transversely polarized nucleon target, $e N^{\uparrow} \rightarrow e \pi X$. In this case the other chiral-odd partner is the Collins fragmentation function, $H_{1}^{\perp}$ [35]. The first evidence of non-trivial transverse spin effects in SIDIS has been observed in the transverse single spin asymmetries measured by the HERMES [36, 37, 38] and the COMPASS [39] experiments where an unpolarized lepton beam is scattered off a transversely polarized proton target, $l p^{\uparrow} \rightarrow l^{\prime} h X$. Besides the non-zero Collins asymmetry, which contains $h_{1}$ and $H_{1}^{\perp}$ discussed previously, another non-zero asymmetry (Sivers asymmetry), was also observed. The Sivers asymmetry is associated with a naive T-odd transverse momentum dependent (TMD) parton distribution function [40].

The first model dependent extraction of the transversity distributions has been carried out [41] by combining SIDIS [38, 37, 42, 43] data with $\mathrm{e}^{+} \mathrm{e}^{-}$collision data [44]. In addition, the extraction of the Sivers function [45, 46, 47, 48, 49] has been performed by combining SIDIS data from the HERMES [38] on the proton and COMPASS data [50] on the deuteron. Complementing the data from the HERMES [38, 37], COMPASS [43], and BELLE [44] experiments, the recent release from the Jefferson Lab Hall A experiment E06-010 [51] on the neutron (with polarized ${ }^{3} \mathrm{He}$ ) will facilitate a flavor decomposition of the transversity distribution function, and the Sivers distribution function in the overlapping kinematic regime. However a model-independent determination of these leading twist functions requires data in a wider kinematic range with high precision in four dimensions $\left(Q^{2}, x, z, P_{T}\right)$, and such an experiment [52] will take place after the $12-\mathrm{GeV}$ energy upgrade at JLab. 


\section{Acknowledgment}

The author thanks Jian-Ping Chen, Latifa Elouadrhiri, Horst Fischer, Achim Geiser, Michel Guidal, M, Huang, K. Krueger, Anna Martin, Dieter Mueller, X. Qian, Emmanuel Sauvan, Bernd Surrow, and C.-P. Yuan for their help in the preparation of this presentation. The author also thanks Qiujian Ye for his help in preparing this manuscript. This work is supported by the U.S. Department of Energy under contract number DE-FG02-03ER41231.

\section{References}

[1] F.D. Aaron et al. (the H1 and ZEUS Collaborations), JHEP01 (2010) 109 [arXiv:0911.0884].

[2] J. Ashman et al.. Phys. Lett., B 206, 364 (1988).

[3] B. W. Filippone and X. D. Ji. Adv. Nucl. Phys., 26, 1 (2001).

[4] S. E. Kuhn, J. P. Chen, and E. Leader. Prog. Part. Nucl. Phys. 63, 1-50 (2009).

[5] M.G. Alekseev et al. (COMPASS Collaboration), arXiv:1007.4061.

[6] V. Y. Alexakhin et al. (COMPASS Collaboration), Phys. Lett. B 647, 8 (2007).

[7] A. Airapetian et al. (Hermes Collaboration), JHEP 08 (2010) 130 [arXiv:1002.3921].

[8] M. Alekseev et al. (COMPASS Collaboration), hep-ex/0802.3023; Phys. Lett. B 676, 31 (2009).

[9] B. Adeva et al. (SMC Collaboration), Phys. Rev. D 70, 012002 (2004).

[10] E. S. Ageev et al. (COMPASS Collaboration), Phys. Lett. B 633, 25 (2006).

[11] M. Stolarski (COMPASS Collaboration), "Measurements of $\frac{\Delta G}{G}$ from high transverse momentum hadrons pairs in COMPASS", in: Proc. of the 16th International Workshop on Deep Inelastic Scattering and QCD (DIS2008), April 7-11, 2008, Londaon, UK, p209.

[12] S. S. Adler et al. (PHENIX Collaboration), Phys. Rev. Lett. 93, 202002 (2004); S. S. Adler et al. (PHENIX Collaboration), Phys. Rev. D 73, 091102 (2006); A. Adare et al. (PHENIX Collaboration), Phys. Rev. D 76, 051106 (2007); A. Adare et al. (PHENIX Collaboration), Phys. Rev. Lett. 103, 012003 (2009); A. Adare et al. (PHENIX Collaboration), Phys. Rev. D 79, 012003 (2009).

[13] B. I. Abelev et al. (STAR Collaboration), Phys. Rev. Lett. 97, 252001 (2006); B. I. Abelev et al. (STAR Collaboration), Phys. Rev. Lett. 100, 232003 (2008).

[14] D. de Florian, R. Sassot, M. Stratmann, W. Vogelsang, Phys. Rev. Lett. 101, 72001 (2008); D. de Florian, R. Sassot, M. Stratmann, W. Vogelsang, Phys. Rev. D 80, 034030 (2009).

[15] J. Blümlein and H.Böttcher, DESY 09-131.

[16] J.C. Collins and D.E. Soper, Phys. Rev. D 16, 2219 (1977); J.P. Ralston and D.E. Soper, Nucl. Phys. B152, 109 (1979); J.C. Collins, D.E. Soper, and G. Sterman, B250, 199 (1985); P.J. Mulders and R.D. Tangerman, Nucl. Phys. B197, 461 (1996); D. Boer and P.J. Mulders, Phys. Rev. D 57, 5780 (1998).

[17] D. Müller et al., Fortsch. Phys. 42, 101 (1994); A. Radyushkin, Phys. Lett. B 385, 333 (1996); Phys. Rev. D 56, 5524 (1997); X. Ji, Phys. Rev. Lett. 78, 610 (1997).

[18] C. Adloff et al. (H1 Collaboration), Phys. Lett. B 57, 417 (2001).

[19] A. Aktas et al. (H1 Collaboration), Eur. Phys. J. C 44, 1 (2005). 
[20] S. Chekanov et al., Phys. Lett. B 573, 46 (2003).

[21] A. Airapetian et al. (HERMES Collaboration), Phys. Rev. Lett. 87, 182001 (2001).

[22] F. Ellinghaus, Nucl. Phys. A 711, 171 (2002).

[23] A. Airapetian et al. (HERMES Collaboration), Phys. Rev. D 75, 011103 (2007).

[24] S. Stepanyan et al. (CLAS Collaboration), Phys. Rev. Lett. 87, 182002 (2001).

[25] F.-X. Girod, R.A. Niyazov et al. (CLAS Collaboration), Phys. Rev. Lett. 100162002 (2008).

[26] S. Chen ret al. (CLAS Collaboration), Phys. Rev. Lett. 97, 072002 (2006).

[27] C. Camacho et al., Phys. Rev. Lett. 97, 262002 (2006).

[28] R. De Masi, M. Garcon, B. Zhao et al. (CLAS Collaboration), Phys. Rev. C 77, 042201(R) (2008).

[29] S.A. Morrow et al. (CLAS Collaboration), Eur. Phys. J. C 39, 5 (2009).

[30] X. Artru and M. Mekhfi. Z. Phys.,C45, 669 (1990).

[31] R. L. Jaffe and X. D. Ji. Phys. Rev. Lett.,67, 552-555 (1991).

[32] P. J. Mulders and R. D. Tangerman. Nucl. Phys., B461, 197-237 (1996).

[33] D. Boer and P. J. Mulders. Phys. Rev., D57, 5780-5786 (1998).

[34] J. P. Ralston and D. E. Soper. Nucl. Phys., B152, 109 (1979); J.L. Cortes et al., Zeit. Phys. C 55, 409 (1992).

[35] J.C. Collins. Nucl. Phys., B396, 161-182 (1993).

[36] A. Airapetian et al., Phys. Lett. B693, 11-16 (2010).

[37] A. Airapetian et al., Phys. Rev. Lett.,03, 152002 (2009).

[38] A. Airapetian et al., Phys. Rev. Lett., 94, 012002 (2005).

[39] M. G. Alekseevet al., arXiv:1005.5609v1 (2010).

[40] D. W. Sivers. Phys. Rev., D41, 83 (1990).

[41] M. Anselmino et al., Phys. Rev., D75, 054032 (2007).

[42] E. S. Ageev et al., Nucl. Phys., B765, 31-70 (2007).

[43] M. Alekseev et al., Phys. Lett., B673, 127-135 (2009).

[44] K. Abe et al., Phys. Rev. Lett., 96, 232002 (2006).

[45] M. Anselmino et al., arXiv:hep-ph/0511017v1 (2005).

[46] M. Anselmino et al., Phys. Rev., D72, 094007 (2005).

[47] M. Anselmino et al., Phys. Rev., D71, 074006 (2005).

[48] J. C. Collins et al., Phys. Rev., D73, 014021 (2006).

[49] M. Anselmino et al., Eur. Phys. J., A39, 89-100 (2009).

[50] V. Y. Alexakhin et al., Phys. Rev. Lett., 94, 202002 (2005).

[51] Jefferson Lab Experiment E06-010, Spokespersons: E. Cisbani, J. P. Chen, H. Gao, X.D. Jiang and J.C. Peng.

[52] H. Gao, L. Gamberg et al., to appear in Eur. Phys. J. Plus 